Published in final edited form as:

Future Neurol. 2009 ; 4(2): 167-177. doi:10.2217/14796708.4.2.167.

\title{
Receptor for advanced glycation end products:
}

\author{
its role in Alzheimer's disease and other neurological diseases
}

Lih-Fen Lue,

Laboratory of Neuroinflammation, Sun Health Research Institute, 10515 West Santa Fe Drive, Sun City, AZ, USA, lihfen.lue@bannerhealth.com

Douglas Gordon Walker, Laboratory of Neuroinflammation, Sun Health Research Institute, 10515 West Santa Fe Drive, Sun City, AZ, USA, douglas.walker@bannerhealth.com

Sandra Jacobson, and

The Cleo Roberts Center, Sun Health Research Institute, 10515 West Santa Fe Drive, Sun City, AZ, USA, sandra.jacobson@bannerhealth.com

\section{Marwan Sabbagh ${ }^{\dagger}$}

The Cleo Roberts Center, Sun Health Research Institute, 10515 West Santa Fe Drive, Sun City, AZ 85351, USA, marwan.sabbagh@bannerhealth.com

\begin{abstract}
The receptor for advanced glycation end products (RAGE) has been demonstrated to play a central role in the pathogenic mechanisms of a growing number of important neurological diseases, including Alzheimer's disease (AD) and stroke. Two functional types of RAGE have been associated with neurological diseases: cell membrane-bound (full length) and soluble. In general, ligand binding to full-length RAGE initiates sustained cellular activation and receptor-dependent signaling resulting in inflammation and cellular stress, and is ultimately associated with increased RAGE expression. By comparison, soluble forms of RAGE, generated either by alternative splicing or by proteolysis, can reduce the severity of the consequence of ligand-membrane RAGE interactions by preventing ligands from binding to the full-length RAGE. This can inhibit the neurotoxic or proinflammatory responses involved in disease states. This article reviews the pathobiology of RAGE, with emphasis on soluble forms of RAGE, and discusses its relevance to AD and to other neurological diseases, as well as how manipulation of the different forms of RAGE is becoming a powerful therapeutic strategy.
\end{abstract}

\section{Keywords}

advanced glycation end products; Alzheimer's disease; amyloid $\beta$ peptide; RAGE; RAGE inhibitors; S100/calgranulins; secreted; transcript variants

\footnotetext{
(C) 2009 Future Medicine

†Author for correspondence: The Cleo Roberts Center, Sun Health Research Institute, 10515 West Santa Fe Drive, Sun City, AZ 85351, USA, Tel.: +1 623875 6500, Fax: +1 623875 6504, marwan.sabbagh@bannerhealth.com

Financial \& competing interests disclosure

The authors have no other relevant affiliations or financial involvement with any organization or entity with a financial interest in or financial conflict with the subject matter or materials discussed in the manuscript apart from those disclosed.

No writing assistance was utilized in the production of this manuscript.
} 
Since the initial isolation and cloning of the receptor for advanced glycation end products (RAGE) in 1992 [1,2], it has emerged as a key molecule playing pivotal roles in the pathogenic mechanisms of several major neurological diseases, including Alzheimer's disease (AD) [3-5], stroke [6,7], multiple sclerosis [8,9], amyotrophic lateral sclerosis [10] and neurological complications of diabetes [11], along with many other peripheral diseases, including cancers, diabetic nephropathy, diabetic retinopathy, atherosclerosis, coronary artery disease and rheumatoid arthritis (recently reviewed [12-14]). In addition to being a pattern-recognition receptor for advanced glycation end products (AGEs), RAGE binds a number of other ligands [15-17]. Prominent among these is the amyloid $\beta(A \beta)$ peptide, which is the central player in AD pathogenic mechanisms [18]. RAGE-associated diseases share common features of the sustained and increased presence of different RAGE ligands in association with disease pathology and the common involvement of vascular dysfunction. Ligand binding activates cell surface-bound full-length RAGE (fIRAGE) leading to inflammatory signaling, which also involves increased expression of RAGE. Different cellular signaling pathways are activated by RAGE ligation, which will be discussed in detail in this review. The central feature is that the $R A G E$ gene promoter contains NF- $\mathrm{KB}$ and SP1 transcription elements that respond to proinflammatory cellular signaling by increased expression of RAGE [19]. The end result of this is that cell-surface RAGE receptor and ligand interaction sets up a positive feedback mechanism that can rapidly accelerate disease progression.

By contrast, soluble forms of RAGE provide significant inhibition to these positive feedback mechanisms, since these forms of RAGE contain functional ligand-binding domains but lack the cellular signaling domains. Their suggested mechanism of action is to bind to RAGE ligands in the extracellular environment, thus preventing the ligand interaction with flRAGE. The therapeutic potential of soluble forms of the RAGE protein was demonstrated in animal model studies of atherosclerosis, diabetes and systemic amyloidosis; all of these studies demonstrated that administration of recombinant soluble RAGE (sRAGE) protein containing the ligandbinding domain had significant disease-modifying effects [20-24].

The discovery of soluble forms of RAGE endogenously present under physiological conditions has provided insight into the protective roles that RAGE molecules could play in these diseases. Over the last few years, as many different alternative forms of RAGE have been identified, the lack of systematic nomenclature has led to a confusing array of different names. Recently, Hudson and colleagues have analyzed 20 RAGE transcripts in lung or aortic smooth muscle cells [24]. Based on their data, they suggested a new unifying naming system for the various RAGE isoforms [24]. As the physiological significance of many of these isoforms is not known, this article will focus on the properties of flRAGE, and the soluble forms of RAGE, generated by alternative splicing (termed endogenous-secretory RAGE [esRAGE]) or enzymaticallycleaved RAGE (ecRAGE) [24-27]. In studies discussed in this review, measurements of sRAGE refer to a pool containing esRAGE and ecRAGE. The carboxyl terminal sequences differ between ecRAGE and esRAGE [28]. Ligand binding with esRAGE and ecRAGE avoids the adverse consequences of activating signal transduction pathways that can increase inflammation and cellular perturbation. As these soluble forms of RAGE compete for ligands with the flRAGE, they can be considered protective.

We will focus on recent findings of how different forms of RAGE are involved with different aspects of $\mathrm{AD}$ pathobiology, and whether they present therapeutic targets for disease modification. 


\section{Transcript variants of the human RAGE gene}

\section{Cell membrane-associated full-length RAGE}

The $R A G E$ gene (gene name: advanced glycosylation end products-specific receptor [AGER]) is located on chromosome 6 within the region of the MHC III [29]. The RAGE gene encodes a protein that belongs to the immunoglobulin (Ig) superfamily of cell surface proteins. Located 22 amino acids from the $\mathrm{N}$-terminal signaling region, the extracellular domain of the fIRAGE protein consists of a single Ig variable region-like domain (V-domain) and two Ig constant region-like domains ( $\mathrm{C} 1$ and $\mathrm{C} 2$ domains) (Figure 1). This is followed by a transmembrane region composed of 21 amino acids, and a cytosolic tail composed of 41 amino acids. The cytosolic tail mediates the signal transduction resulting from binding of ligands to the extracellular domains [24].

\section{Alternative splice variants}

Alternative splicing is a post-transcriptional modification, generated at the pre-mRNA level that allows a single gene to code more than one form of the protein. The $R A G E$ gene can produce transcripts that are alternatively spliced in multiple ways, yielding a variety of potential protein variants that are expressed by a wide variety of cell types [28,30-33]. The resulting proteins from these transcripts have different properties, including the absence of ligand-binding sites (i.e., N-terminal truncated), and the absence of the transmembrane domain with a distinct $\mathrm{C}$ terminal sequence from 332 to 347 (esRAGE).

Recently, Hudson et al. characterized the RAGE alternative splice variants in human lung and cultured aortic smooth muscle cells [24]. They identified 19 RAGE variants, including the three previously-documented variants termed esRAGE, N-truncated RAGE and $\Delta^{8}$-RAGE, which were designated with a newly revised nomenclature system as RAGE_v1, RAGE_v2 and RAGE_v3, ten newly identified variants (RAGE_v4 to RAGE_v13) and six other previously reported spliced variants (RAGE_v14 to RAGE_v19) that were not detected in lung tissues or smooth muscle cells, but were identified by others. Among these variants, RAGE_v1, which lacks the transmembrane domain and possesses a unique C-terminal sequence, was demonstrated to be secreted by cultured aortic smooth muscle cells [24]. Membrane-bound fIRAGE is the more abundant form in human lung and aortic smooth muscle cells compared with RAGE_v1. The RAGE_v1 expression level was only $1 / 11$ in human lung and 1/7 in human aortic smooth muscle cells of flRAGE [24].

\section{RAGE variants in human brain}

With the exception of lung tissues where constitutive expression of fIRAGE is abundant, RAGE is expressed at low levels in most other tissues, including normal human brain tissue. Expression in the brain appears to increase with aging and in $\mathrm{AD}[34,35]$. Characterization of the alternative splice variants in human brains revealed that the brain soluble form of RAGE variants shared the same sequence with vascular cells, astrocytes and peripheral mononuclear cells [31]. However, in contrast to lung tissues and smooth muscle cells where the most a bundantly expressed form of RAGE is full length, the most abundant form of RAGE in the hippocampus is RAGE_v1 (previously designated as esRAGE and sRAGE), at levels approximately fourfold higher than the flRAGE at RNA levels [31]. This does seem to indicate that the brain has cellular mechanisms to prevent uncontrolled RAGE-mediated inflammation. This mechanism must be deficient in diseases such as AD. Most of the studies on RAGE transcript variants have been at the mRNA level. Further studies of soluble forms of RAGE proteins within tissue are needed. Another soluble form of RAGE transcript (designated hRAGEsec) was identified in the human brain by in situ hybrization. In contrast to flRAGE, hRAGEsec was detected more intensely in hippocampal CA3 pyramidal cells, dentate gyrus granule cells and cortical neurons, as well as in glial cells in white matter [32]. Sequence 
analysis demonstrated that this soluble form of RAGE was different from esRAGE. Its physiological significance has been questioned by others [24,28].

Since the binding of RAGE ligands to RAGE_v1 or flRAGE leads to opposite consequences, further understanding of how alternative splicing is regulated in the healthy and diseased brain is important and may provide opportunity for the therapeutic manipulation of these systems.

\section{RAGE involvement in AD pathology: human studies}

With the finding that RAGE could be activated by $A \beta$ peptide, its significance in the pathogenic mechanism of AD became clear [18,34,36-38]. We previously characterized RAGE expression in the hippocampus, inferior frontal cortex and cerebellum in postmortem $\mathrm{AD}$ and nondemented controls [34]. A significant elevation of total RAGE level in AD was detected in the pathology-enriched regions (hippocampus and inferior frontal cortex) when compared with the same regions in the nondemented controls. We also demonstrated that levels of RAGE proteins positively correlated with the severity of the AD pathology [34]. Consistent with biochemical analysis, immunohistochemical procedures using antibodies against the extracellular region of the RAGE molecules detected increased RAGE immunoreactivity in neurons, microglia and astrocytes in AD-pathological regions. The antibody used in the study preferentially identified flRAGE. This was confirmed in another study using an antibody to RAGE that recognized fIRAGE and RAGE with a molecular weight of $35 \mathrm{kD}$, which showed strongly increased RAGE staining in astrocytes and neurons in AD brains [39]. A study using an antibody specific for esRAGE found differential changes in this protein in astrocytes and neurons in AD hippocampus regions Cornus Ammonis (CA)1 and CA3. There was a significant reduction of esRAGE immunoreactivity in neurons, but increased expression in astrocytes [40]. These studies indicate that AD pathology alters the expressions of different forms of RAGE in cell type- and brain region-dependent manners. RAGE expression in human AD cerebral vessels has become a subject of interest since it has been demonstrated that RAGE can mediate transport of $A \beta$ across the blood-brain barrier (BBB) $[4,41]$. It was reported that there was a progressive increase in staining of cerebral blood vessels for RAGE protein with increasing severity of AD pathology [42,43]. Increased expression of RAGE on cerebral blood vessels could lead to enhanced passage of $A \beta$ into the vessels and brains from the circulation $[44,45]$. We have found that the ability to stain for RAGE in cerebral blood vessels is dependent on the antibodies being used. In our earlier work, cerebral vessels did not stain prominently for RAGE, while in later studies we found that the immunoreactivity was more apparent using an antibody raised against full-length human recombinant RAGE [LuE $L F, \mathrm{~W}_{\text {ALKER }} \mathrm{D}$,

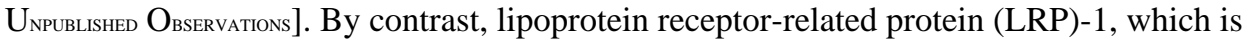
expressed on the abluminal surface of cerebral endothelial cells, transports $A \beta$ from the brain to the blood. LRP-1 levels are decreased during aging and in $\mathrm{AD}$, thus reducing $\mathrm{A} \beta$ transport out of the brain and promoting its accumulation in the neuropil $[42,43,46]$.

\section{Multiple roles for RAGE in AD pathology: transgenic mouse studies}

Since RAGE has been demonstrated to be involved in multiple AD-related pathological processes, transgenic mouse models have been developed that allow investigation of mechanisms of RAGE interactions with $\mathrm{A} \beta$ and how this can induce pathology. In one study using amyloid plaque-developing transgenic mice $\mathrm{Tg} 2576$, it was demonstrated that infusion of $A \beta$ into the blood resulted in RAGE-mediated $A \beta$ transport into the brain [41]. Uptake of $A \beta$ was blocked using an antibody to RAGE that was infused into the mice with the $A \beta$; the antibody bound to cerebral vessels expressing RAGE, thus preventing endothelial RAGE and $A \beta$ interactions. The same effect was observed if mice were infused with recombinant sRAGE protein along with the A $\beta$. This was the first indication that the soluble form of the RAGE protein might have a therapeutic use in $\mathrm{AD}$ [41]. This same study demonstrated that 
administration of recombinant sRAGE to plaque-developing mice for 6 months significantly reduced the accumulation of $A \beta$ in the brain [41]. $A \beta$ bound to recombinant sRAGE could be detected in the circulation of treated mice. This study was of particular interest as it established that $A \beta$ from the circulation can significantly contribute to $A \beta$ accumulations in the brain. In a different transgenic mouse model, double transgenic mice that overexpressed neuronal human fIRAGE and mutated human amyloid precursor protein (APP) developed enhanced neuropathology and enhanced deposition of $A \beta$ during aging compared with single transgenic APP mice [47]. It was demonstrated that these double transgenic animals had enhanced activation of NF- $\mathrm{kB}$ due to RAGE-A $\beta$ interactions, which resulted in significantly higher levels of microgliosis and astrocytosis, as well as significantly enhanced decreases in long-term potentiation and ability to perform memory tasks. Evidence for neurotoxicity in this mouse model was detected at 3-4 months of age, even before there was significant $A \beta$ deposition [47]. In a recent study, oligomeric $A \beta$-mediated inhibition of long-term potentiation in the entorhinal cortex was not observed when tested in tissue slices from RAGE-null mice or dominant-negative RAGE mice (the RAGE transgene did not contain the intracellular signal transduction domain) [48]. In this study, it was demonstrated that RAGE-A $\beta$ interactions induced p 38 MAPK activation. Inhibiting p 38 activation reduced the A $\beta$-mediated impairment of long-term potentiation, while inhibiting the activation of JNK had no effect [48].

\section{Involvement of additional RAGE ligands in dementia \& other neurological disorders}

The major focus of RAGE in AD has been its interaction with A $\beta$; however, RAGE activation can also be contributed by other ligands that are induced during the disease process. Southern et al. found a correlation between the level of cognitive impairment and the intensity of staining for Ne-(carboxymethyl)-lysine, the most abundant AGE, in neurons and vessels of those with vascular dementia [49]. AGE-modified tau is present in neurofibrillary tangles in AD, while glyceraldehyde-modified AGEs are increased in the cytoplasm of neurons in the hippocampus and parahippocampal gyrus of AD cases [50,51]. These toxic AGEs can induce cell death when applied to neurons [52]. Some of the calgranulin family members (e.g., S100B, S100A9 and S100A12), which are RAGE ligands, can be produced by activated microglia and astrocytes, and their levels are increased in AD brains in association with plaques [53]. These molecules can amplify oxidative stress and inflammatory responses through interaction with RAGE. The contributions of these ligands to the disease could depend on their concentrations, types of cells responding and the local cellular environment where they are accumulated [54,55]. Currently, the effects of all RAGE ligands are not completely understood. For example, treating cultured LAN-5 human neuronal-like cells with low concentrations of S100B $(0.5-500 \mathrm{nM})$ protected them from $A \beta$-mediated toxicity through a RAGE-mediated mechanism, but higher doses of S100B enhanced A $\beta$ toxicity. S100B increased RAGE expression on these cells in a dosedependent manner [55].

The RAGE-mediated mechanisms in different neurological diseases may be determined by the predominant ligands; for examples, high-mobility group box 1 (HMGB1) is the major ligand in stroke/ischemia, AGE-modified protein in diabetic retinopathy and $A \beta$ in AD. However, there are overlaps between the ligands and diseases. In $\mathrm{AD}$ brains, although $\mathrm{A} \beta$ is the major ligand, AGE-modified proteins and S100B are also elevated [49,53]. Overall effects of the RAGE mechanisms on a specific disease may be dependent on the profiles of the RAGE ligands.

\section{RAGE-A $\beta$ interactions}

Biochemical studies have demonstrated that sRAGE interactions with A $\beta$ peptide strongly inhibited its aggregation [56]. These data have suggested that soluble forms of RAGE in the 
circulation could not only sequester $\mathrm{A} \beta$, preventing its passage into the brain, but could also reduce cell-membrane perturbation caused by $A \beta$ oligomerization at the cell surface.

\section{RAGE involvement in cerebral ischemia}

The role of RAGE in mediating tissue damage in cerebral ischemia is becoming apparent from human and transgenic mouse studies. An increase in the RAGE ligand HMGB1 occurs in the circulation of stroke patients and is released from the site of ischemia in a mouse model. Ischemic brain damage could be reduced if mice were treated with an antibody to HMGB1, an antagonist of HMGB1 or with sRAGE protein [6]. The mechanism of damage appeared to be due to inflammatory activation of RAGE-expressing microglia by HMGB1. In another study, it was demonstrated that there was a rapid release of HMGB1 from neurons following ischemia; this was accompanied by a rapid increase in RAGE expression in the peri-infarct region [7]. In vitro studies demonstrated that HMGB1 had potent proinflammatory activation properties on astrocytes and endothelial cells, inducing expression of TNF- $\kappa$ and intercellular adhesion molecule-1 [7].

\section{RAGE \& diabetic brain complications}

It has been well established that chronic diabetes causes damage to the periphery, including kidneys, retina, peripheral nerves and, in particular, blood vessels, through RAGE-mediated mechanisms [14,57-63]. However, the brain is also affected, probably secondary to vascular pathology. Chronic diabetes can cause cerebral atrophy, white matter loss and cognitive decline, although Type 2 diabetes appears to have greater consequences than Type $1[64,65]$. Using a mouse model of chronic diabetes, animals developed white matter lesions, cerebral atrophy and cognitive dysfunction, similar to that observed in human diabetics. It was demonstrated that at the sites of white matter lesions, there was strong induction of RAGE expression that colocalized with expression of myelin basic protein [66]. These animals also had increased expression of RAGE throughout the gray matter on neurons, astrocytes, microglia and vessels, and increased expression on oligodendrocytes. By comparison, RAGEnull mice that were also made diabetic for the same periods of time did not show the same degree of cerebral atrophy or white matter loss as wild-type mice. These findings indicate that diabetic neurological complications are mediated, in part, by RAGE $[11,66]$.

\section{Signaling of RAGE-ligand interaction}

Engagement of flRAGE by a ligand triggers activation of key cell-signaling pathways, such as p2 $1^{\text {ras }}$, MAPKs, NF- $\kappa B$ and cdc42/rac [67]. Activation of some pathways is dependent on the ligand, but activation of the transcription factor NF- $\kappa \mathrm{B}$ following ligand interaction with RAGE is a common feature of RAGE signaling. Other studies have indicated that activation of p38 MAPK and ERK1 and 2 MAPK occurs at the same time or precedes NF- $\mathrm{KB}$ activation $[68,69]$. This was observed in two cancer cell lines activated with S100A8/A9 or S100P [68, 70]. It was observed that AGE-induced collagen synthesis in NRK-49F cells mediated by RAGE activation resulted in increased phosphorylation of STAT- 1 and -3 transcription factors [71]. Activation of neuronal hybrid cells by $A \beta$ resulted in activation of $\mathrm{p} 38$, JNK pathways and NF- $\mathrm{KB}$ with reduced cellular viability; this effect was blocked by treatment of cells with RAGE-blocking antibodies [72]. As these key cellular-signaling pathways are required for normal cellular function, the feasibility of preventing RAGE activation through cellular signaling is yet to be determined.

\section{Soluble RAGE as a product of proteolysis}

As soluble forms of RAGE could have therapeutic potential, this brings us back to the issue of the nature of sRAGE. For example, ELISAs specific for esRAGE only measure a portion 
of the sRAGE pool in biofluids or cell culture-conditioned medium when compared with an ELISA using two RAGE antibodies with epitopes in the extracellular domain, which should detect all soluble forms of RAGE. Studies using plasma of diabetic and normal patients detected esRAGE levels at approximately 17-34\% of total sRAGE levels [73,74]. Measurement of plasma of healthy adults demonstrated that the circulating RAGE concentration was five-times higher than esRAGE concentration. These findings suggested that additional mechanisms other than alternative splicing are involved in the generation of soluble forms of RAGE in the circulation.

Recent in vitro studies identified enzymes capable of cleaving flRAGE, thus releasing it from the plasma membranes $[27,75,76]$. These enzymes are matrix metalloproteinase 9 and/or a disintegrin and metalloprotease (ADAM)-10. ADAM-10, similar to ADAM-17, are shed through ectodomain shedding to regulate the functions of membrane-bound receptors. As mentioned previously, we will distinguish RAGE_v1 (sRAGE or esRAGE) generated by alternative splicing from released RAGE by designating the latter as 'ecRAGE' (representing the enzymatically cleaved, soluble form of RAGE).

The site of enzyme cleavage appears to be nine amino acids above the plasma membrane, which generates an $\mathrm{N}$-terminal fragment with intact ligand-binding domain and a $\mathrm{C}$-terminal fragment $(12 \mathrm{kDa})$ that remains cell-associated (Figure 1). The N-terminal fragment, ecRAGE (51 kDa), was slightly larger than alternatively spliced esRAGE (48 kDa) when comparing gel electrophoresis samples of media from HEK cells either transfected with HEK fIRAGE or with esRAGE [27]. The C-terminal fragment appeared to be a substrate for $\gamma$-secretase, the enzyme complex responsible for releasing the C-terminal of A $\beta$ peptide from the APP [76].

Similar to other Ig superfamily members, cleavage and release of membrane-bound flRAGE molecules appears to be the main mechanism for generating circulating sRAGE. Evidence suggested that only plasma membrane-bound fIRAGE is susceptible to proteolysis. Shedding of flRAGE does not appear to significantly alter the levels of cell-associated RAGE level, suggesting that cleavage only affects a small part of the total cellular RAGE pool [27]. Studies were mainly carried out on gene-transfected cells, so these findings raise questions concerning the major physiological sources of circulating ecRAGE [27,75,76]. In addition, the physiological and/or pathological factors that regulate shedding of flRAGE in vivo must also be determined. The enzymes shown to contribute to the formation of ecRAGE are ubiquitous and expressed in most tissues and by many different cell types.

It appears that RAGE shedding can occur under constitutive and inducible conditions, but how is this process affected by disease-specific factors? Elevated levels of sRAGE correlate with reduced risk for coronary artery disease, hypertension, metabolic syndrome, arthritis and AD, amongst others [77-87]. Different disease states are associated with reduced levels of sRAGE; does this mean that the disease mechanisms are directly responsible for reducing RAGE shedding? This is an important question that could have significant therapeutic potential, particularly in the brain, on how to increase endogenous sRAGE levels by increasing enzymatic cleavage.

\section{Soluble RAGE as a predictive marker for disease protection}

In the past 2-3 years, there have been a growing number of studies demonstrating that levels of sRAGE in the circulation are protective against a range of diseases and even promote longevity. Interestingly, studies have demonstrated that centenarians had significantly higher levels of circulating sRAGE than a group of young controls [88]. Similarly, in a group of disease-free controls, it was demonstrated that circulating sRAGE levels negatively correlate with body mass index and waist:hip ratio (known risk factors for diabetes, vascular diseases 
and AD), supporting a possible protective role for these proteins before any evidence of diabetic or vascular complications has occurred [89].

Two studies have demonstrated that there were significantly lower levels of circulating sRAGE in AD patients compared with cognitively normal controls [79] as well as between patients with early cognitive decline and normal controls [81], suggesting that RAGE is involved early in the pathogenesis of AD. The fact that sRAGE levels are declining in the circulation along with progressive cognitive decline in AD strongly suggests that circulating sRAGE and RAGE ligands (e.g., $A \beta$ in the circulation) are influencing pathological events in the brain. These findings support the hypothesis that circulating $A \beta$ is contributing to the formation of cerebral plaques, but it needs to be remembered that other RAGE ligands, such as S100A8, A9 and A12, are also present in the circulation at s ignificant levels [53].

\section{Potential effects of using soluble RAGE mimetics as a therapeutic approach to modify AD}

Recent studies have found that RAGE levels decreased with the progression of cognitive decline in AD subjects, indicating that restoring circulating sRAGE to normal levels could be useful for slowing down disease progression. The potential beneficial effects of addition of bioactive forms of sRAGE mimetics in blood are illustrated in Figure 2. First, peripheral levels of RAGE ligands, such as S100/calgranulins, AGEs and HMGB1, can be reduced, which can ultimately attenuate vascular inflammation and increase integrity of the BBB. Second, by increasing circulating sRAGE levels, more $A \beta$ peptide can be scavenged from the peripheral $A \beta$ pool. This can prevent $A \beta$ trafficking across the BBB to the brain. Third, since $A \beta$ from the peripheral pool is reduced, more $A \beta$ can be drawn out of the brain. This may eventually reduce brain amyloid load, inflammatory responses and adverse consequences due to the activation of flRAGE.

\section{Intervention of RAGE activation in AD}

RAGE inhibitors are being developed as treatments for AD. Two potential approaches to this are developing therapeutics based on SRAGE and those that can inhibit activation of RAGE cellular signaling. Recently, Phase I and IIa safety studies were completed for the RAGE antagonist PF-04494700, formerly known as TTP488 (Pfizer, NY, USA and TransTech Pharma, NC, USA). This agent is an orally bioavailable small molecule antagonist of RAGE, which appears to have good safety and tolerability in human subjects, and is undergoing dose finding and efficacy studies (Phase II) [ $\left.\mathrm{S}_{\text {ABBAGGH }} \mathrm{M}, \mathrm{U}_{\mathrm{NPUBLISHED}} \mathrm{D}_{\mathrm{ATA}}\right]$. As this drug is under commercial development, there are no peer reviewed scientific studies, but the company website states that this agent was effective in lowering $A \beta$ load in amyloid-plaque developing transgenic mice [101]. This company is also developing a sRAGE fusion protein (designated TTP4000) for use in anti-RAGE therapy.

\section{Conclusion}

The receptor for advanced glycation end products has emerged as a key molecule that plays pivotal roles in the pathogenic mechanisms in several major diseases, including diabetes, atherosclerosis, arthritis, cancer and AD. Many of these diseases have vascular pathological components in which RAGE also plays a prominent role. Its significance as a disease-causing mechanism may be due to its activation by a broad range of disease-associated ligands. Although normal functions for RAGE activation have been described, most studies only identify pathological involvement of RAGE activation. This suggests that blocking RAGE activation in adults is a reasonable therapeutic strategy that may not have significant side effects. Similar to many proinflammatory receptors, soluble forms of RAGE are produced by 
cells; this appears to be a naturally occurring negative-feedback mechanism to prevent uncontrolled RAGE-mediated inflammation and cellular stress. Since reduction of circulating RAGE levels correlates significantly with disease states in a significant number of diseases, this provides good evidence that not only is RAGE involved in the pathogenesis of these diseases, but that soluble forms of RAGE also have significant protective properties.

\section{Future perspective}

Given the growing understanding of RAGE and how it relates to the pathobiology of AD, diabetes and other diseases, some of the important questions to be answered are:

- What is the mechanism responsible for reducing levels of circulating sRAGE in AD and other diseases?

- Is it simply that the sRAGE has been cleared after complexing with higher levels of circulating ligands?

- Does the pathological mechanism directly decrease the cellular production of sRAGE?

- Are both of these mechanisms responsible?

With current understanding of RAGE mechanisms, the interest in further development of RAGE inhibitors as new therapeutic agents will increase. Recent studies identifying mechanisms of cleavage of flRAGE from the cellular surface open up significant new therapeutic strategies. It will be useful to administer agents that increase the rate that fIRAGE is removed from the plasma membrane to increase the levels of circulating RAGE. As cellular cleavage and shedding appears to contribute to a more significant extent than secreted RAGE generated by alternative splicing, this may be a potential avenue for developing RAGE-based therapeutics for the brain, where larger molecules cannot penetrate. Based on the studies of recombinant sRAGE in animal models, adding sRAGE mimetics into the biofluids may also have potential to become a therapeutic strategy for AD.

\section{Executive summary}

- The receptor for advanced glycation end products (RAGE) has been demonstrated to play a central role in pathogenic mechanisms of a growing number of important neurological and other diseases, including Alzheimer's disease (AD), atherosclerosis and diabetes.

- In general, ligand binding to RAGE initiates cellular activation and receptordependent signaling. Sustained elevation of ligand levels leads to a positivefeedback loop that ultimately results in increased RAGE expression and ligand binding.

- Two functional types of RAGE have been described: membrane-bound and soluble.

- Soluble forms of RAGE do not have the cellular-signaling domain, but most have the ligand-binding domain. This means that they can sequester the ligand, thus preventing it from binding to full-length RAGE (fIRAGE) and activating what are generally seen as damaging proinflammatory pathways.

- Complex patterns of alternative RAGE transcription have been identified with the potential to generate up to 19 different forms of RAGE mRNA, which can produce proteins with a range of different properties. 
- Soluble forms of RAGE generated by alternative splicing (designated endogenoussecretory RAGE) or proteolysis (designated enzymatically-cleaved RAGE) can reduce the severity of ligand-membrane RAGE interactions by preventing ligands from binding to the cell surface flRAGE. This inhibits the neurotoxic, proinflammatory or profibrotic responses involved in disease states, such as AD, atherosclerosis and diabetes.

- The major pathway for the production of circulating RAGE appears to be due to enzymatic cleavage of fIRAGE from the plasma membrane by the enzymes ADAM-10 and MMP-9.

- Given the growing understanding of RAGE and how it relates to the pathobiology of $\mathrm{AD}$, diabetes and other diseases, there will be increased interest in cultivating RAGE inhibitors or soluble RAGE mimetics as new therapeutic approaches to these diseases.

- Circulating levels of soluble RAGE correlate with the progression of several RAGE-mediated diseases, including AD. Whether monitoring the changes of soluble RAGE levels can be useful as an indicator for disease development and severity requires further investigation.

\section{Acknowledgments}

This work is supported by funding from the National Institute of Neurological Disorders and Stroke (RO1 NS049075 to LFL), Alzheimer's Association (IIRG-08-91996 to LFL), and the National Institute on Aging to the Arizona Alzheimer's disease Core Center (P30 AG 019610).

\section{Bibliography}

Papers of special note have been highlighted as:

- of interest

•• of considerable interest

1. Schmidt AM, Vianna M, Gerlach M, et al. Isolation and characterization of two binding proteins for advanced glycosylation end products from bovine lung which are present on the endothelial cell surface. J. Biol. Chem 1992;267(21):14987-14997. [PubMed: 1321822]• Original description of receptor for advanced glycation end products (RAGE) isolation and characterization as receptor for the ligand, advanced glycation end products, in biological tissues.

2. Neeper M, Schmidt AM, Brett J, et al. Cloning and expression of a cell surface receptor for advanced glycosylation end products of proteins. J. Biol. Chem 1992;267(21):14998-15004. [PubMed: 1378843]• Cloned for the first time the human lung RAGE gene.

3. Chen X, Walker DG, Schmidt AM, Arancio O, Lue LF, Yan SD. RAGE: a potential target for A $\beta$ mediated cellular perturbation in Alzheimer's disease. Curr. Mol. Med 2007;7(8):735-742. [PubMed: 18331231]

4. Deane R, Wu Z, Zlokovic BV. RAGE (yin) versus LRP (yang) balance regulates Alzheimer amyloid $\beta$-peptide clearance through transport across the blood-brain barrier. Stroke 2004;35(11 Suppl 1): 2628-2631. [PubMed: 15459432]

5. Maczurek A, Shanmugam K, Munch G. Inflammation and the redox-sensitive AGE-RAGE pathway as a therapeutic target in Alzheimer's disease. Ann. NY Acad. Sci 2008;1126:147-151. [PubMed: 18448809]

6. Muhammad S, Barakat W, Stoyanov S, et al. The HMGB1 receptor RAGE mediates ischemic brain damage. J. Neurosci 2008;28(46):12023-12031. [PubMed: 19005067] 
7. Qiu J, Nishimura M, Wang Y, et al. Early release of HMGB-1 from neurons after the onset of brain ischemia. J. Cereb. Blood Flow Metab 2008;28(5):927-938. [PubMed: 18000511]

8. Yan SS, Wu ZY, Zhang HP, et al. Suppression of experimental autoimmune encephalomyelitis by selective blockade of encephalitogenic T-cell infiltration of the central nervous system. Nat. Med 2003;9(3):287-293. [PubMed: 12598893]

9. Qin J, Goswami R, Dawson S, Dawson G. Expression of the receptor for advanced glycation end products in oligodendrocytes in response to oxidative stress. J. Neurosci. Res 2008;86(11):2414-2422. [PubMed: 18438937]

10. Iłzecka J. Serum-soluble receptor for advanced glycation endproducts levels in patients with amytrophic lateral sclerosis. Acta Neurol. Scand. 2008DOI: 10.1111/J. 1600-0404.2008.011.33.xEpub ahead of print

11. Toth C, Martinez J, Zochodne DW. RAGE, diabetes, and the nervous system. Curr. Mol. Med 2007;7 (8):766-776. [PubMed: 18331235]

12. Clynes R, Moser B, Yan SF, Ramasamy R, Herold K, Schmidt AM. Receptor for AGE (RAGE), weaving tangled webs within the inflammatory response. Curr. Mol. Med 2007;7(8):743-751. [PubMed: 18331232]

13. Logsdon CD, Fuentes MK, Huang EH, Arumugam T. RAGE and RAGE ligands in cancer. Curr. Mol. Med 2007;7(8):777-789. [PubMed: 18331236]

14. Yamamoto H, Watanabe T, Yamamoto Y, et al. RAGE in diabetic nephropathy. Curr. Mol. Med 2007;7(8):752-757. [PubMed: 18331233]

15. Hofmann MA, Drury S, Fu C, et al. RAGE mediates a novel proinflammatory axis: a central cell surface receptor for S100/calgranulin polypeptides. Cell 1999;97(7):889-901. [PubMed: 10399917]

16. Huttunen HJ, Kuja-Panula J, Sorci G, Agneletti AL, Donato R, Rauvala H. Coregulation of neurite outgrowth and cell survival by amphoterin and S100 proteins through receptor for advanced glycation end products (RAGE) activation. J. Biol. Chem 2000;275(51):40096-40105. [PubMed: 11007787]

17. Wautier JL, Zoukourian C, Chappey O, et al. Receptor-mediated endothelial cell dysfunction in diabetic vasculopathy. Soluble receptor for advanced glycation end products blocks hyperpermeability in diabetic rats. J. Clin. Invest 1996;97(1):238-243. [PubMed: 8550841]•• One of the early reports supporting the protective effects of recombinant soluble RAGE in diabetic animal model.

18. Yan SD, Chen X, Fu J, et al. RAGE and amyloid- $\beta$ peptide neurotoxicity in Alzheimer's disease. Nature 1996;382(6593):685-691. [PubMed: 8751438]•• Provides mechanistic evidence for how RAGE and amyloid $\beta(A \beta)$ interaction could be pathogenic in Alzheimer's disease (AD).

19. Li J, Schmidt AM. Characterization and functional analysis of the promoter of RAGE, the receptor for advanced glycation end products. J. Biol. Chem 1997;272(26):16498-16506. [PubMed: 9195959]

20. Bucciarelli LG, Wendt T, Qu W, et al. RAGE blockade stabilizes established atherosclerosis in diabetic apolipoprotein E-null mice. Circulation 2002;106(22):2827-2835. [PubMed: 12451010]

21. Goova MT, Li J, Kislinger T, et al. Blockade of receptor for advanced glycation endproducts restores effective wound healing in diabetic mice. Am. J. Pathol 2001;159(2):513-525. [PubMed: 11485910]

22. Yan SD, Zhu H, Zhu A, et al. Receptor-dependent cell stress and amyloid accumulation in systemic amyloidosis. Nat. Med 2000;6(6):643-651. [PubMed: 10835680]

23. Wautier JL, Zoukourian C, Chappey O, et al. Receptor-mediated endothelial cell dysfunction in diabetic vasculopathy. Soluble receptor for advanced glycation end products blocks hyperpermeability in diabetic rats. J. Clin. Invest 1996;97(1):238-243. [PubMed: 8550841]

24. Hudson BI, Carter AM, Harja E, et al. Identification, classification, and expression of RAGE gene splice variants. FASEB J 2008;22(5):1572-1580. [PubMed: 18089847]•• Proposes a new nomenclature system for RAGE alternative splice variants.

25. Cheng C, Tsuneyama K, Kominami R, et al. Expression profiling of endogenous secretory receptor for advanced glycation end products in human organs. Mod. Pathol 2005;18(10):1385-1396. [PubMed: 15933755]

26. Raucci A, Cugusi S, Antonelli A, et al. A soluble form of the receptor for advanced glycation endproducts (RAGE) is produced by proteolytic cleavage of the membrane-bound form by the sheddase a disintegrin and metalloprotease 10 (ADAM10). FASEB J 2008;22(10):3716-3727. [PubMed: 18603587] 
27. Zhang L, Bukulin M, Kojro E, et al. Receptor for advanced glycation end products is subjected to protein ectodomain shedding by metalloproteinases. J. Biol. Chem 2008;283(51):35507-35516. [PubMed: 18952609]

28. Yonekura H, Yamamoto Y, Sakurai S, et al. Novel splice variants of the receptor for advanced glycation end-products expressed in human vascular endothelial cells and pericytes, and their putative roles in diabetes-induced vascular injury. Biochem. J 2003;370(Pt 3):1097-1109. [PubMed: 12495433]•• Identifies and names the major alternative splice variant esRAGE.

29. Sugaya K, Fukagawa T, Matsumoto K, et al. Three genes in the human MHC class III region near the junction with the class II: gene for receptor of advanced glycosylation end products, PBX2 homeobox gene and a notch homolog, human counterpart of mouse mammary tumor gene int-3. Genomics 1994;23(2):408-419. [PubMed: 7835890]

30. Park IH, Yeon SI, Youn JH, et al. Expression of a novel secreted splice variant of the receptor for advanced glycation end products (RAGE) in human brain astrocytes and peripheral blood mononuclear cells. Mol. Immunol 2004;40(16):1203-1211. [PubMed: 15104125]

31. Ding Q, Keller JN. Splice variants of the receptor for advanced glycosylation end products (RAGE) in human brain. Neurosci. Lett 2005;373(1):67-72. [PubMed: 15555779]

32. Malherbe P, Richards JG, Gaillard H, et al. cDNA cloning of a novel secreted isoform of the human receptor for advanced glycation end products and characterization of cells co-expressing cell-surface scavenger receptors and Swedish mutant amyloid precursor protein. Brain Res. Mol. Brain Res 1999;71(2):159-170. [PubMed: 10521570]

33. Schlueter C, Hauke S, Flohr AM, Rogalla P, Bullerdiek J. Tissue-specific expression patterns of the RAGE receptor and its soluble forms - a result of regulated alternative splicing? Biochim. Biophys. Acta 2003;1630(1):1-6. [PubMed: 14580673]

34. Lue LF, Walker DG, Brachova L, et al. Involvement of microglial receptor for advanced glycation endproducts (RAGE) in Alzheimer's disease: identification of a cellular activation mechanism. Exp. Neurol 2001;171(1):29-45. [PubMed: 11520119]• Provides evidence that A $\beta$ and RAGE interaction in human microglia-mediated persistent inflammatory response in AD.

35. Jeynes B, Provias J. Evidence for altered expression in Alzheimer lesion pathogenesis. Curr. Alzheimer Res 2008;5(5):432-437. [PubMed: 18855584]

36. Yan SD, Zhu H, Fu J, et al. Amyloid- $\beta$ peptide-receptor for advanced glycation endproduct interaction elicits neuronal expression of macrophage-colony stimulating factor: a proinflammatory pathway in Alzheimer disease. Proc. Natl Acad. Sci. USA 1997;94(10):5296-5301. [PubMed: 9144231]

37. Yan SD, Stern D, Kane MD, Kuo YM, Lampert HC, Roher AE. RAGE-A $\beta$ interactions in the pathophysiology of Alzheimer's disease. Restor. Neurol. Neurosci 1998;12(23):167-173. [PubMed: 12671312]

38. Yan SD, Roher A, Chaney M, Zlokovic B, Schmidt AM, Stern D. Cellular cofactors potentiating induction of stress and cytotoxicity by amyloid $\beta$-peptide. Biochim. Biophys. Acta 2000;1502(1): 145-157. [PubMed: 10899440]

39. Sasaki N, Toki S, Chowei H, et al. Immunohistochemical distribution of the receptor for advanced glycation end products in neurons and astrocytes in Alzheimer's disease. Brain Res 2001;888(2): 256-262. [PubMed: 11150482]

40. Nozaki I, Watanabe T, Kawaguchi M, et al. Reduced expression of endogenous secretory receptor for advanced glycation endproducts in hippocampal neurons of Alzheimer's disease brains. Arch. Histol. Cytol 2007;70(5):279-290. [PubMed: 18431028]

41. Deane R, Du YS, Submamaryan RK, et al. RAGE mediates amyloid- $\beta$ peptide transport across the blood-brain barrier and accumulation in brain. Nat. Med 2003;9(7):907-913. [PubMed: 12808450] -• Provides evidence for the concept that endothelial RAGE molecules are responsible for the influx of circulating $A \beta$ to the brain.

42. Miller MC, Tavares R, Johanson CE, et al. Hippocampal RAGE immunoreactivity in early and advanced Alzheimer's disease. Brain Res 2008;1230:273-280. [PubMed: 18657529]

43. Donahue JE, Flaherty SL, Johanson CE, et al. RAGE, LRP-1, and amyloid- $\beta$ protein in Alzheimer's disease. Acta Neuropathol 2006;112(4):405-415. [PubMed: 16865397] 
44. Mackic JB, Stins M, McComb JG, et al. Human blood-brain barrier receptors for Alzheimer's amyloid- $\beta$ 1- 40. Asymmetrical binding, endocytosis, and transcytosis at the apical side of brain microvascular endothelial cell monolayer. J. Clin. Invest 1998;102(4):734-743. [PubMed: 9710442]

45. Deane R, Zlokovic BV. Role of the blood-brain barrier in the pathogenesis of Alzheimer's disease. Curr. Alzheimer Res 2007;4(2):191-197. [PubMed: 17430246]

46. Deane R, Sagare A, Zlokovic BV. The role of the cell surface LRP and soluble LRP in blood-brain barrier A $\beta$ clearance in Alzheimer's disease. Curr. Pharm. Des 2008;14(16):1601-1605. [PubMed: 18673201]

47. Arancio O, Zhang HP, Chen X, et al. RAGE potentiates A $\beta$-induced perturbation of neuronal function in transgenic mice. EMBO J 2004;23(20):4096-4105. [PubMed: 15457210]•• Demonstrates strong evidence in the amyloid precursor protein and RAGE double transgenic mice that RAGE and A $\beta$ interaction leads to neurodegeneration and learning deficiency.

48. Origlia N, Righi M, Capsoni S, et al. Receptor for advanced glycation end product-dependent activation of p38 mitogen-activated protein kinase contributes to amyloid- $\beta$-mediated cortical synaptic dysfunction. J. Neurosci 2008;28(13):3521-3530. [PubMed: 18367618]

49. Southern L, Williams J, Esiri MM. Immunohistochemical study of N-E-carboxymethyl lysine (CML) in human brain: relation to vascular dementia. BMC Neurol 2007;7:35. [PubMed: 17939855]

50. Choei H, Sasaki N, Takeuchi M, et al. Glyceraldehyde-derived advanced glycation end products in Alzheimer's disease. Acta Neuropathol 2004;108(3):189-193. [PubMed: 15221334]

51. Ko LW, Ko EC, Nacharaju P, et al. An immunochemical study on tau glycation in paired helical filaments. Brain Res 1999;830(2):301-313. [PubMed: 10366687]

52. Sato T, Shimogaito N, Wu X, Kikuchi S, Yamagishi S, Takeuchi M. Toxic advanced glycation end products (TAGE) theory in Alzheimer's disease. Am. J. Alzheimers Dis. Other Demen 2006;21(3): 197-208. [PubMed: 16869341]

53. Shepherd CE, Goyette J, Utter V, et al. Inflammatory S100A9 and S100A12 proteins in Alzheimer's disease. Neurobiol. Aging 2006;27(11):1554-1563. [PubMed: 16253391]

54. Donato R. RAGE: a single receptor for several ligands and different cellular responses: the case of certain S100 proteins. Curr. Mol. Med 2007;7(8):711-724. [PubMed: 18331229]

55. Businaro R, Leone S, Fabrizi C, et al. S100B protects LAN-5 neuroblastoma cells against A $\beta$ amyloidinduced neurotoxicity via RAGE engagement at low doses but increases $A \beta$ amyloid neurotoxicity at high doses. J. Neurosci. Res 2006;83(5):897-906. [PubMed: 16477616]

56. Chaney MO, Stine WB, Kokjohn TA, et al. RAGE and amyloid $\beta$ interactions: atomic force microscopy and molecular modeling. Biochim. Biophys. Acta 2005;1741(12):199-205. [PubMed: 15882940]• Uses molecular modeling to predict the interaction sites of soluble RAGE molecules with $A \beta$.

57. Hudson BI, Wendt T, Bucciarelli LG, et al. Diabetic vascular disease: it's all the RAGE. Antioxid. Redox Signal 2005;7(1112):1588-1600. [PubMed: 16356122]

58. Hudson BI, Schmidt AM. RAGE: a novel target for drug intervention in diabetic vascular disease. Pharm. Res 2004;21(7):1079-1086. [PubMed: 15290845]

59. Kim W, Hudson BI, Moser B, et al. Receptor for advanced glycation end products and its ligands: a journey from the complications of diabetes to its pathogenesis. Ann. NY Acad. Sci 2005;1043:553561. [PubMed: 16037278]

60. Myint KM, Yamamoto Y, Sakurai S, et al. Blockade of diabetic vascular injury by controlling of AGE-RAGE system. Curr. Drug Targets 2005;6(4):447-452. [PubMed: 16026264]

61. Sakurai S, Yonekura H, Yamamoto Y, et al. The AGE-RAGE system and diabetic nephropathy. J. Am. Soc. Nephrol 2003;14(8 Suppl 3):S259-S263. [PubMed: 12874443]

62. Wendt T, Tanji N, Guo J, et al. Glucose, glycation, and RAGE: implications for amplification of cellular dysfunction in diabetic nephropathy. J. Am. Soc. Nephrol 2003;14(5):1383-1395. [PubMed: 12707408]

63. Yan SF, Ramasamy R, Bucciarelli LG, et al. RAGE and its ligands: a lasting memory in diabetic complications? Diab. Vasc. Dis. Res 2004;1(1):10-20. [PubMed: 16305050]

64. Jongen C, van der Grand J, Kappelle LJ, Biessels GJ, Viergever MA, Pluim JP. Automated measurement of brain and white matter lesion volume in Type 2 diabetes mellitus. Diabetologia 2007;50(7):1509-1516. [PubMed: 17492428] 
65. Brands AM, Biessels GJ, Kappelle LJ, et al. Cognitive functioning and brain MRI in patients with Type 1 and Type 2 diabetes mellitus: a comparative study. Dement. Geriatr. Cogn. Disord 2007;23 (5):343-350. [PubMed: 17374953]

66. Toth C, Schmidt AM, Tuor UI, et al. Diabetes, leukoencephalopathy and RAGE. Neurobiol. Dis 2006;23(2):445-461. [PubMed: 16815028]

67. Huttunen HJ, Fages C, Rauvala H. Receptor for advanced glycation end products (RAGE)-mediated neurite outgrowth and activation of NF- $\mathrm{KB}$ require the cytoplasmic domain of the receptor but different downstream signaling pathways. J. Biol. Chem 1999;274(28):19919-19924. [PubMed: 10391939]

68. Hermani A, De SB, Medunjanin S, Tessier PA, Mayer D. S100A8 and S100A9 activate MAP kinase and NF- $\kappa \mathrm{B}$ signaling pathways and trigger translocation of RAGE in human prostate cancer cells. Exp. Cell Res 2006;312(2):184-197. [PubMed: 16297907]

69. Yeh CH, Sturgis L, Haidacher J, et al. Requirement for p38 and p44/p42 mitogen-activated protein kinases in RAGE-mediated nuclear factor- $\mathrm{kB}$ transcriptional activation and cytokine secretion. Diabetes 2001;50(6):1495-1504. [PubMed: 11375353]

70. Fuentes MK, Nigavekar SS, Arumugam T, et al. RAGE activation by S100P in colon cancer stimulates growth, migration, and cell signaling pathways. Dis. Colon Rectum 2007;50(8):1230-1240. [PubMed: 17587138]

71. Huang JS, Guh JY, Chen HC, Hung WC, Lai YH, Chuang LY. Role of receptor for advanced glycation end-product (RAGE) and the JAK/STAT-signaling pathway in AGE-induced collagen production in NRK-49F cells. J. Cell Biochem 2001;81(1):102-113. [PubMed: 11180401]

72. Onyango IG, Tuttle JB, Bennett JP Jr. Altered intracellular signaling and reduced viability of Alzheimer's disease neuronal cybrids is reproduced by $\beta$-amyloid peptide acting through receptor for advanced glycation end products (RAGE). Mol. Cell. Neurosci 2005;29(2):333-343. [PubMed: 15911356]

73. Humpert PM, Djuric Z, Kopf S, et al. Soluble RAGE but not endogenous secretory RAGE is associated with albuminuria in patients with Type 2 diabetes. Cardiovasc. Diabetol 2007;6:9. [PubMed: 17343760]

74. Katakami N, Matsuhisa M, Kaneto H, et al. Endogenous secretory RAGE but not soluble RAGE is associated with carotid atherosclerosis in Type 1 diabetes patients. Diab. Vasc. Dis. Res 2008;5(3): 190-197. [PubMed: 18777492]

75. Raucci A, Cugusi S, Antonelli A, et al. A soluble form of the receptor for advanced glycation endproducts (RAGE) is produced by proteolytic cleavage of the membrane-bound form by the sheddase a disintegrin and metalloprotease 10 (ADAM10). FASEB J 2008;22(10):3716-3727. [PubMed: 18603587]

76. Galichet A, Weibel M, Heizmann CW. Calcium-regulated intramembrane proteolysis of the RAGE receptor. Biochem. Biophys. Res. Commun 2008;370(1):1-5. [PubMed: 18355449]•• First to report that soluble form of RAGE can be generated by ADAM10 from the full-length RAGE.

77. Bopp C, Hofer S, Weitz J, et al. sRAGE is elevated in septic patients and associated with patients outcome. J. Surg. Res 2008;147(1):79-83. [PubMed: 17981300]

78. Choi KM, Yoo HJ, Kim HY, et al. Association between endogenous secretory RAGE, inflammatory markers and arterial stiffness. Int. J. Cardiol 2009;132(1):96-101. [PubMed: 18190981]

79. Emanuele E, D'Angelo A, Tomaino C, et al. Circulating levels of soluble receptor for advanced glycation end products in Alzheimer disease and vascular dementia. Arch. Neurol 2005;62(11):17341736. [PubMed: 16286548]•• Provides evidence that circulating soluble RAGE level is reduced in AD patients.

80. Geroldi D, Falcone C, Emanuele E. Soluble receptor for advanced glycation end products: from disease marker to potential therapeutic target. Curr. Med. Chem 2006;13(17):1971-1978. [PubMed: 16842191]

81. Ghidoni R, Benussi L, Glionna M, et al. Decreased plasma levels of soluble receptor for advanced glycation end products in mild cognitive impairment. J. Neural. Transm 2008;115(7):1047-1050. [PubMed: 18506386] 
82. Katakami N, Matsuhisa M, Kaneto H, et al. Endogenous secretory RAGE but not soluble RAGE is associated with carotid atherosclerosis in Type 1 diabetes patients. Diab. Vasc. Dis. Res 2008;5(3): 190-197. [PubMed: 18777492]

83. Moser B, Hudson BI, Schmidt AM. Soluble RAGE: a hot new biomarker for the hot joint? Arthritis Res. Ther 2005;7(4):142-144. [PubMed: 15987496]

84. Nakamura K, Yamagishi S, Adachi H, et al. Serum levels of sRAGE, the soluble form of receptor for advanced glycation end products, are associated with inflammatory markers in patients with Type 2 diabetes. Mol. Med 2007;13(34):185-189. [PubMed: 17592553]

85. Sternberg Z, Weinstock-Guttman B, Hojnacki D, et al. Soluble receptor for advanced glycation end products in multiple sclerosis: a potential marker of disease severity. Mult. Scler 2008;14(6):759763. [PubMed: 18505774]

86. Stewart C, Cha S, Caudle RM, Berg K, Katz J. Decreased levels of soluble receptor for advanced glycation end products in patients with primary Sjogren's syndrome. Rheumatol. Int 2008;28(8): 771-776. [PubMed: 18231794]

87. Zhang H, Tasaka S, Shiraishi Y, et al. Role of soluble receptor for advanced glycation end products on endotoxin-induced lung injury. Am. J. Respir. Crit. Care Med 2008;178(4):356-362. [PubMed: 18535257]

88. Geroldi D, Falcone C, Minoretti P, Emanuele E, Arra M, D'Angelo A. High levels of soluble receptor for advanced glycation end products may be a marker of extreme longevity in humans. J. Am. Geriatr. Soc 2006;54(7):1149-1150. [PubMed: 16866696]

89. Norata GD, Garlaschelli K, Grigore L, et al. Circulating soluble receptor for advanced glycation end products is inversely associated with body mass index and waist/hip ratio in the general population. Nutr. Metab. Cardiovasc. Dis. 2008DOI: 10.1016/j. numecd.2008.03.004Epub ahead of print

101. WebsiteTransTech PharmaTTP488, Alzheimer's

Diseasewww.ttpharma.com/pipeline_rage_antagonists.html 


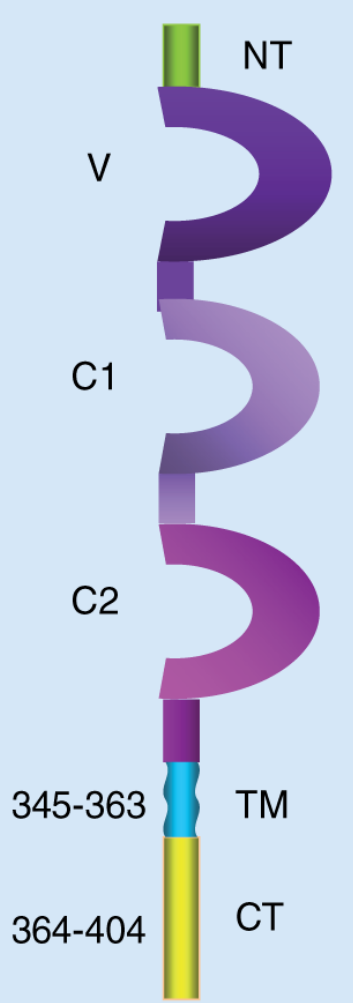

fIRAGE

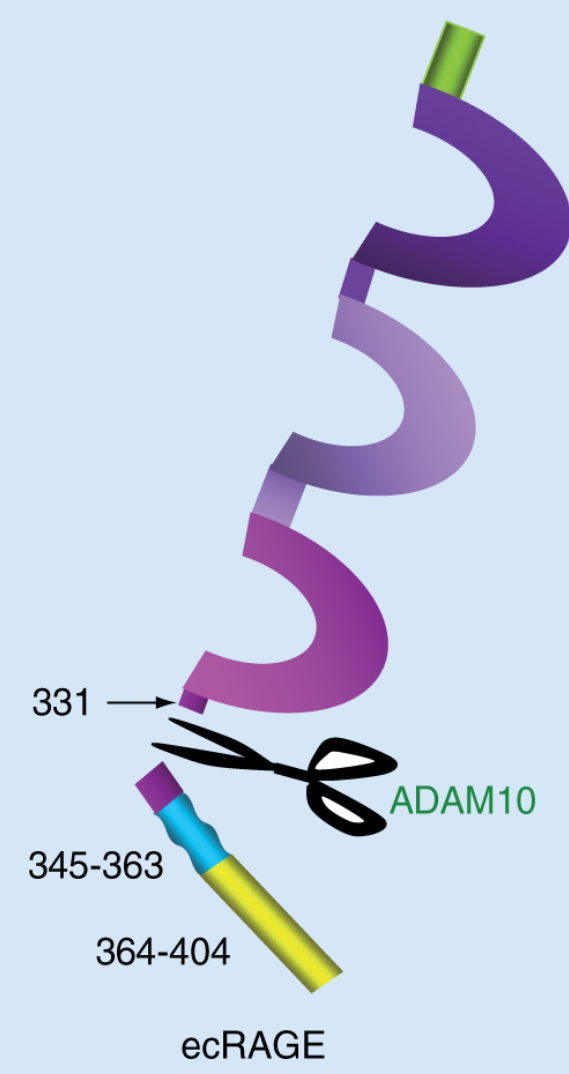

Alternative-splice RAGE_v1 (esRAGE)

Figure 1. Three major forms of RAGE in neurological diseases

In this figure, the 404 amino acid, flRAGE consists of N-terminal (NT), variable (V), constant $\mathrm{C} 1$ and C2 extracellular regions, a transmembrane domain (TM), and a cytosolic terminal (CT). Soluble ecRAGE is generated by the activity of ADAM10, which presumably cleaves between amino acid 331 and 332, leaving a CT fragment as a potential substrate for $\gamma$-secretase. The alternative-splice RAGE_v1 (also termed esRAGE) in humans consists of 347 amino acids, including a distinct CT sequence from amino acids 332 to 347.

ecRAGE: Enzymatically cleaved RAGE; flRAGE: Full-length RAGE; RAGE: Receptor for advanced glycation end products. 


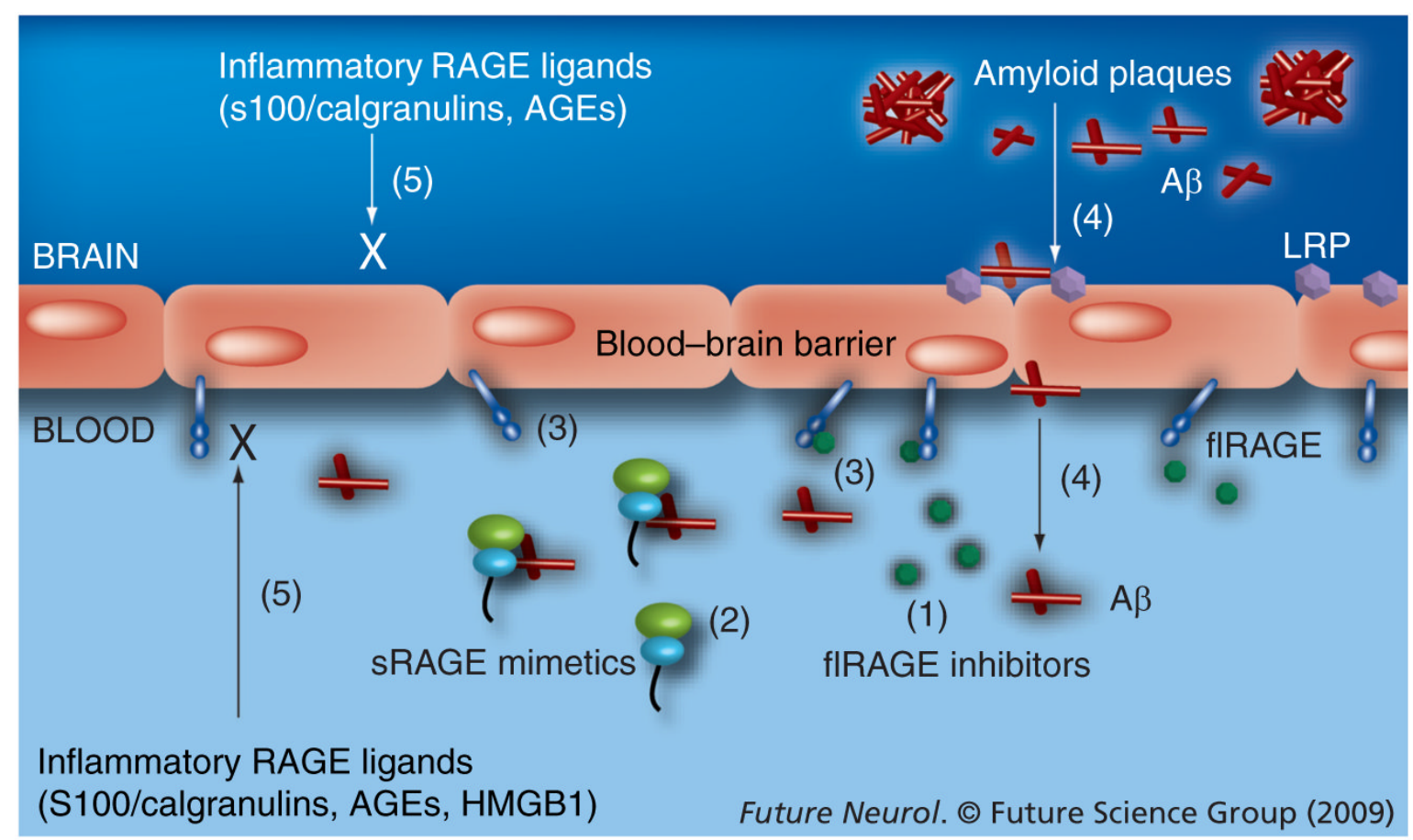

Figure 2. Potential strategy to prevent RAGE activation in Alzheimer's disease blood-brain barrier This figure suggests that adding (1) RAGE inhibitors or (2) sRAGE mimetics into the circulation will (3) reduce flRAGE activation by endothelial cells at the blood-brain barrier and (4) promote $A \beta$ transport from brain to the periphery by LRP-1. This will reduce the effects of other RAGE inflammatory ligands (S100/calgranulins and AGEs) in the brain and circulation (5). A $\beta$ : Amyloid $\beta$; AGE: Advanced glycation end product; HMGB1: Highmobility group box 1; LRP: Low-density lipoprotein-related protein; sRAGE: Soluble receptor for advanced glycation end product. 